\title{
Henkel hat Finanzziele 2012 erreicht
}

Henkel ist es 2012 gelungen, den Gesamtumsatz auf 16.510 Mio. Euro und damit um 5,8 Prozent gegenüber dem Vorjahr zu steigern. Organisch, das heißt bereinigt um Wechselkurseffekte und Akquisitionen/Divestments, stieg der Umsatz um 3,8 Prozent. Dieses Wachstum war sowohl preis- als auch mengengetrieben. Zum organischen Umsatzwachstum trugen alle Unternehmensbereiche bei - so auch Adhesive Technologies.

O bwohl sich die Konjunktur im Jahresverlauf abschwächte, konnte der Unternehmensbereich Adhesive Technologies im vergangenen Jahr seinen profitablen Wachstumskurs fortsetzen und den Umsatz um 3,6 Prozent auf den bisherigen Höchstwert von 8.256 Mio. Euro steigern.

Unternehmensangaben zufolge trug vor allem die konsequente Ausrichtung des Portfolios auf innovative Kundenlösungen zu dieser guten Entwicklung bei.

Insbesondere in den Wachstumsregionen zog der Umsatz stark an. Das größte Plus erzielte die Region Afrika/ Nahost. Hier legte der Umsatz zweistellig zu. In den reifen Märkten wurde insgesamt eine positive Umsatzentwicklung erzielt, vor allem dank der starken Entwicklung in Nordamerika.
Diese glich die negativen wirtschaftlichen Rahmenbedingungen in Westeuropa, insbesondere in den südeuropäischen Ländern, aus.

Das bereinigte betriebliche Ergebnis stieg um 15,9 Prozent und erreichte mit 1.246 Mio. Euro einen neuen Höchstwert. Die bereinigte Umsatzrendite wuchs um 1,2 Prozentpunkte und erzielte mit 15,1 Prozent ebenfalls einen neuen Höchstwert. Das betriebliche Ergebnis stieg um 18,9 Prozent auf 1.191 Mio. Euro.

„Wir erwarten für das Geschäftsjahr 2013 ein organisches Umsatzwachstum zwischen 3 und 5 Prozent. Für die bereinigte EBIT-Marge rechnen wir mit einem Anstieg auf etwa 14,5 Prozent und für das bereinigte Ergebnis je Vorzugsaktie mit einem Zuwachs von etwa 10 Prozent“, sagte Kasper Rorsted,

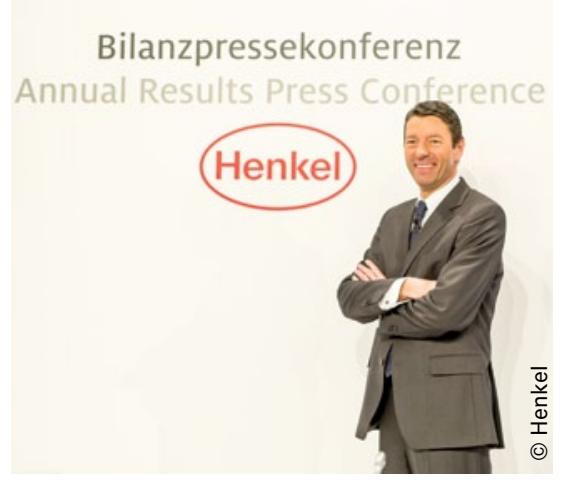

Kasper Rorsted, Vorsitzender des Henkel-Vorstands: „Wir erwarten für das Geschäftsjahr 2013 ein organisches Umsatzwachstum zwischen 3 und 5 Prozent.“

Vorsitzender des Henkel-Vorstands bei der Vorstellung des Geschäftsberichts 2012.

\section{Neue Erkenntnisse auf dem Gebiet ionischer Flüssigkeiten}

Der Rostocker Chemiker Prof. Dr. Udo Kragl wurde kürzlich im Rahmen des Strategieprozesses Biotechnologie 2020+ für seine innovativen Ansätze zur Kombination von ionischen Flüssigkeiten und Biokatalyse mit einem Forschungspreis des Bundesministeriums für Bildung und Forschung (BMBF) ausgezeichnet. Ionische Flüssigkeiten könnten auch für die Herstellung neuartiger Klebstoffe interessant sein.

W ährend die Biokatalyse seit Jahrzehnten wegen der selektiven und effizienten Reaktionsbedingungen im Bereich der Herstellung von Feinchemikalien und Pharmabausteinen fest etabliert ist, haben sich ionische Flüssigkeiten als neuartige Klasse von Lösungsmitteln erst in der letzten Dekade eine Reihe von neuen Anwendungsfeldern erschlossen“, erläutert Prof. Kragl, Lehrstuhlinhaber für Technische Chemie und Dekan der Interdisziplinären Fakultät der Universität Rostock. Ihre speziellen Eigenschaften ermögliche eine spezielle Kombination von darin gelösten Stoffen, zum Beispiel für die Stabilisierung von Enzymen als Biokatalysatoren oder als selektives Extraktionsmittel für Biomoleküle. Die Anwendungsgebiete ionischer Flüssigkeiten seien schier unbegrenzt. Sie können bei der Zellulosespaltung oder Optimierung von Schmiermitteln eingesetzt werden, ebenso aber auch für neuartige Klebstoffe oder in der Medizin. Verbunden mit dem Preis ist eine Projektförderung in Höhe von 1,8 Millionen Euro über die nächsten fünf Jahre.

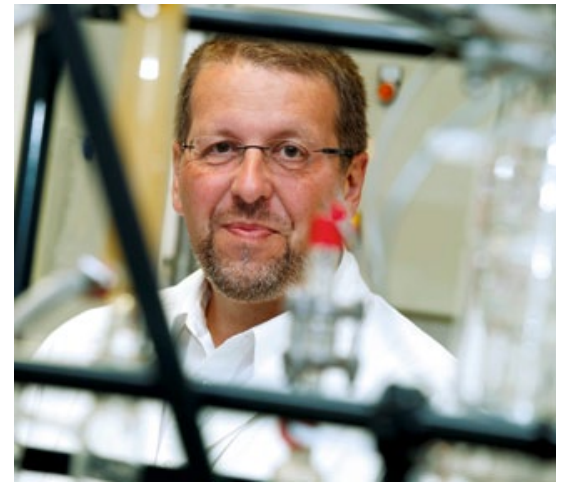

Prof. Kragl, Lehrstuhlinhaber für Technische Chemie und Dekan der Interdisziplinären Fakultät der Universität Rostock 